\title{
Competencias docentes y conductas disruptivas: Un desafío permanente en el contexto educativo
}

Teaching competences and disruptive behaviors: a permanent challenge in the educational context.

Gisela Tatiana Alonso Delgado. ${ }^{1}$, Marilyn Jessenia Paredes Argudo. ${ }^{2}$ \& Giancarlo Daniel Salazar Olaya. ${ }^{3}$

\begin{abstract}
.
DOI: https://doi.org/10.33262/cienciadigital.v4i4.1.1450

The educational reality worldwide and also in Ecuador, unlike in previous years, is currently becoming more inclusive, participatory and diverse. However, the search to find timely responses to the diversity of cases that attend the different educational units and levels of education continues to be a great challenge. In this order, one of the problems of greatest concern related to the development of teaching competencies in order to comprehensively attend to students with the presence of disruptive behaviors. In observation made in the Adalberto Ortiz Quiñonez Educational Unit, marked limitations could be detected in the teachers who work with the 8th year students of Basic General Education with disruptive behaviors, which prevents them from being properly cared for. In line with what has been stated, the objective of our work is focused on: Analyzing teaching competencies and their impact on the attention of students with disruptive behaviors. Based on a mixed methodology, of a non-experimental descriptive type, supported by different techniques such as focus group, the survey, the interview and the observation guide, the main result is the confirmation of the idea to defend, which led to the design of a Manual of playful activities that allowed teachers to be provided with a series of activities and games of great utility for the attention of disruptive behaviors in 8th year students, favoring the development of their teaching competences and the compensation of behavior problems of students object of study.
\end{abstract}

Key words: Teaching skills, disruptive behaviors, manual, game.

\footnotetext{
${ }^{1}$ Unidad Educativa Fiscal Fermín Vera Rojas, gisella1812@ hotmail.com

${ }^{2}$ Centro Educativo Naciones Unidad, jesseniaaparedes@gmail.com

${ }^{3}$ Hospital General IESS. Milagro. giandaniel18@ hotmail.com
} 


\section{Resumen}

La realidad educativa a nivel mundial y también en Ecuador a diferencia de años anteriores se torna actualmente más incluyente, participativa y diversa. Sin embargo la búsqueda por encontrar respuestas oportunas ante la diversidad de casos que asisten a las diferentes unidades educativas y niveles de enseñanza sigue constituyendo un gran desafío. En este orden uno de los problemas que más preocupa está relacionado con el desarrollo de competencias docentes para poder atender de manera integral los estudiantes con presencia de conductas disruptivas. En observación realizada en la Unidad Educativa Adalberto Ortiz Quiñonez se pudo detectar marcadas limitaciones en los docentes que laboran con los estudiantes de 8vo año de Educación General Básica con conductas disruptivas, lo cual impide que sean atendidos adecuadamente. En correspondencia con lo señalado el objetivo de nuestro trabajo se centra en: Analizar las competencias docentes y su incidencia en la atención de los estudiantes con conductas disruptivas. Basada en una metodología mixta, de tipo descriptiva no experimental, apoyada en diferentes técnicas como grupo focal, la encuesta, la entrevista y la guía de observación, se logra como principal resultado la confirmación de la idea a defender, lo cual derivó en el diseño de un Manual de actividades lúdicas que permitió propiciar a los docentes una serie de actividades y juegos de gran utilidad para la atención de conductas disruptivas en los estudiantes de 8vo año, favoreciendo el desarrollo de sus competencias docentes y la compensación de los problemas de conducta de los estudiantes objeto de estudio.

Palabras clave: Competencias docentes, conductas disruptivas, manual, juego.

\section{Introducción.}

La realidad educativa a nivel mundial a diferencia de años anteriores se torna actualmente más incluyente, participativa y diversa. Sin embargo, tanto en Latinoamérica como en Ecuador a pesar de los avances logrados la búsqueda por encontrar respuestas oportunas ante la diversidad de casos que asisten a las diferentes Unidades Educativas y niveles de enseñanza sigue constituyendo un gran desafío.

Si bien es cierto que con el transcurrir histórico se ha ido creando la necesidad de buscar estrategias y alternativas educativas que abran espacios a una verdadera inclusión y atención a la diversidad, misma que debe estar sustentada en una visión más amplia y humana; también compartimos que a pesar de los múltiples esfuerzos realizados aún no se logra alcanzar los estándares deseados de calidad en lo que a inclusión se refiere.

En este orden de ideas resulta importante destacar que uno de los problemas más preocupantes en este momento en el contexto educativo ecuatoriano, está precisamente relacionado con los altos índices de estudiantes que presentan conductas disruptivas; muchos de los cuales se originan desde el propio manejo familiar, acompañado de una insuficiente estrategia de intervención educativa y social. 
Por otra parte los impactos que trae en los procesos de enseñanza-aprendizaje y en todo lo relacionado con la formación, la inclusión educativa y social de los casos de niños, adolescentes y jóvenes con manifestaciones de conductas disruptivas simples o complejas, además de ser una gran preocupación constituye un gran reto para el claustro de docentes que demandan tener competencias acordes para poder atender la diversidad de estudiantes y a su vez dar respuestas precisas a los casos con conductas disruptivas desde una visión multidisciplinar y sistemática.

Como es lógico pensar lo explicado implica el desarrollo de procesos de intervención mucho más organizados e intencionados que incluyen la participación e implicación del contexto educativo, pero también del socio -familiar donde se desenvuelve el menor. Para esto el docente debe desarrollar una gran suma de competencias que le faciliten asumir con responsabilidad los retos de la educación y la enseñanza frente al comportamiento conductual de los estudiantes.

En observación realizada en la Unidad Educativa “Adalberto Ortiz Quiñonez" ubicada en la Cooperativa. Nueva Prosperina de la ciudad de Guayaquil, se pudo detectar la presencia en los salones de clases del 8vo nivel de enseñanza básica de varios casos de estudiantes que de manera reiterada manifiestan conductas disruptivas tales como: agresión a los compañeros, inadecuadas actitudes con los profesores y demás estudiantes, interrupciones a los docentes, no cumplimiento de reglas, incumplimiento en la realización de los deberes escolares, entre otras manifestaciones. Teniendo en cuenta lo descrito los estudiantes son víctimas de comentarios negativos y lo que es peor de exclusiones tanto por parte de los docentes como del resto de los estudiantes.

Ante estas situaciones se pudo apreciar que no siempre el grupo de docentes contaba con las herramientas y competencias pedagógicas y didácticas necesarias para responder oportunamente a las demandas educativas de estos casos, trayendo serios problemas tanto para el propio estudiante como para los docentes y familiares. La problemática se agudiza debido a que los docentes carecen de preparación profesional relacionada a actividades específicas donde se les oriente cómo atender conductas disruptivas desde su desempeño docente. Es por ello que nos preguntemos ¿Cómo inciden las competencias docentes en la atención a estudiantes con conductas disruptivas de 8vo año de educación general básica en la Unidad Educativa Adalberto Ortiz Quiñonez? Para dar respuesta a nuestro problema de investigación nos plantemos como Objetivo General: Analizar las competencias docentes y su incidencia en la atención de los estudiantes con conductas disruptivas.

\section{De los orígenes a la actualidad sobre las conductas disruptivas.}

Los estudios de Cuberes (2013), al citar a (Céspedes, 2012), explican que se estima que entre 3 y 5 de cada 10 niños y adolescentes muestran conductas desafiantes, de carácter ocasional, de los cuales 2 de cada 10 niños exhiben conductas oposicionistas de forma habitual. 
Según (Sanders y Hendry, 1997, citados por Calvo 2003, Cuberes, 2013 \& Flores, 2018), .. "La disrupción es un término que procede del inglés Disruptive y que se utiliza para nombrar aquello que produce una ruptura brusca." Enfatizan los autores que:... "La conducta disruptiva es cualquier conducta que entorpece el orden y la disciplina en la escuela y el bienestar educativo de los alumnos..."

Los autores precedentes explican que dentro de las manifestaciones más comunes se encuentran la desobediencia, interrupciones, impuntualidades, ruidos extremos, incumplimientos, desajustes emocionales y del comportamiento; todo lo cual influye de manera negativa en el proceso de enseñanza-aprendizaje y en el desempeño normal del educando.

Lo cierto es que desde la propia existencia del hombre, han existido personas con diferentes y diversos comportamientos, que según la época social han sido o no aceptados, muchas veces dependiendo del criterio que la propia sociedad ha ido imponiendo. Es por ello que la literatura consultada habla indistintamente de trastornos de conductas, trastornos del comportamiento, conductas desafiantes, conductas negativas, inadaptados sociales entre otros términos, hasta llegar a definirlas como: conductas disruptivas.

Según indica Freire (2012),... "Los comportamientos disruptivos son procederes constantes plasmados por el escolar para obstaculizar la tarea del educador y de los compañeros, propiciando un mal clima en el aula, acumulando sus actuaciones inadecuadas que tienen como intención perturbar el entorno del salo de clase."... concluye el autor y comenta que los docentes no siempre utilizan métodos activadores de enseñanza donde permiten al estudiante el desarrollo de un aprendizaje significativo con características de tipo motivadora.

Posteriormente en el 2015, Jurado, explica que es muy frecuente encontrar jóvenes que presentan dificultades para integrarse en los institutos educativos, identificándose procesos de inadaptación en los cuales se manifiestan comportamientos disruptivos. Este mismo autor señala que por lo general las conductas disruptivas de los estudiantes afectan los procesos de enseñanza- aprendizaje, enfatizando en la necesidad de establecer la relación entre conductas disruptivas, fracaso escolar y los procesos de intervención para favorecer la capacidad resiliente de los estudiantes y el desarrollo de aprendizajes significativos en los diferentes niveles de enseñanza.

Otra de las fuentes consultadas evidencian que: ... "las conductas disruptivas, son acciones perturbadoras o agresivas que rompen la disciplina y alteran la armonía del grupo dificultando el proceso de enseñanza-aprendizaje y la atención en el aula. http://educaryaprender.es/conductas-disruptivas-comportamiento/

A nuestra consideración se trata de conductas que generan básicamente desajustes y conflictos tanto en el aula como socialmente, cuyas manifestaciones están relacionadas con causas internas y externas que motivan diversos trastornos del comportamiento, constituyendo un desafío permanente para los docentes los padres de familia y todo el entorno que rodea al menor. 


\section{Formas de intervención y atención, su relación con las competencias docentes.}

Un aspecto muy preocupante en torno a las formas de intervención y escolarización de los estudiantes con conductas disruptivas y que nos hace coincidir con (Flores, 2018), es precisamente que en muchas ocasiones ante la presencia de discentes con conductas disruptivas en el contexto escolar además de no contarse con todas las herramientas y competencias para su atención también estos sufren las consecuencias derivadas de sus desajustes comportamentales, de ahí que sean excluidos tanto por sus compañeros como por parte del docente, afectándose notablemente sus posibilidades de aprendizaje y de incorporación a la vida activa.

En este orden las consideraciones de Jiménez, (2017), resultan interesantes sobre todo cuando indica que tanto el desempeño docente como las propias competencias funcionales a tener en cuenta están establecidas en las normas educativas, donde la planeación, el tiempo y el uso de estrategia variadas, son en extremo importantes, sin embargo estas pueden estar afectados por la disrupción en el aula, lo que genera un intenso nivel de estrés en el docente, unido a disminución en los indicadores de eficiencia del trabajo docente.

Otro de los criterios que se deben tener en cuenta cuando nos planteamos procesos de intervención y atención a niños, adolescentes o jóvenes con conductas disruptivas según refieren (Jurado, \& Justiano, 2015), es la necesidad de que todo proceso de intervención debe iniciar por atender las causas que lo provocan y los cambios existentes en los entornos educativos, en los procesos psicosociales y biológicos de los menores y sobre todo tener un carácter sistémico y sistemático.

A los análisis anteriores, los autores del presente trabajo concuerdan en añadir que todo proceso de intervención a realizar debe estar concebido desde la multidisciplinaridad y la responsabilidad compartida de todos los participantes, donde el medio familiar también juega un rol esencial en la compensación de los estados conductuales de sus hijos. En correspondencia con ello, consideramos que la escuela y todo el ente educativo que recibe el menor deben contar con todas las competencias y condiciones requeridas para poder intervenir oportunamente no sólo en detectar la presencia de conductas disruptivas, sino también para poder enfocar adecuadamente los procesos de orientación e intervención.

Ahora bien, cabe entonces preguntarnos: Cómo lograr desarrollar competencias docentes para poder atender la diversidad manifiesta en cada grupo clase?

Para dar respuesta a la interrogante anterior lo primero sería definir ¿qué entendemos por competencias?, La palabra competencia viene del latín competencia que tiene dos acepciones: a) competencia, cf. competir, que significa: disputa o contienda entre dos o más personas sobre algo; b) competencia, competente, que significa: incumbencia; pericia, aptitud, idoneidad para hacer algo o intervenir en un asunto determinado, el significado de competencia se puede asociar con el sustantivo griego dinámis que significa: fuerza, capacidad, habilidad, talento y poder (Expasa, 2018). 
Se puede entender por competencia, las aptitudes que poseen una persona; es decir, las capacidades, habilidades y destreza con las que cuenta para realizar una actividad determinada o para tratar un tema específico de la mejor manera posible.

Desde el punto de vista profesional según, Duarte (2017), es una persona con elevada preparación, competencia y especialización que presta un servicio público, proporcionándole cierto de grado de privilegio, autoridad y reconocimiento social.

Todo ello nos hace coincidir con la literatura consultada en que una persona es competente en la medida que posea conocimientos teóricos y habilidades prácticas, motivos, valores y actitudes que le permitan dar solución a las disímiles situaciones que puede enfrentar tanto desde el punto de vista personal como profesional de manera lógica y eficaz.

En un segundo momento y entrando en el terreno específico de las competencias docentes, resulta imperante mencionar que para que él o la docente ejerza su profesión con éxito requiere además del conocimiento permanente y progresivo de su disciplina, debe disponer de un verdadero manejo didáctico de la misma.

En correspondencia con lo planteado se asume lo señalado por Morazán (2013) en su estudio sobre Competencias docentes y su relación con el rendimiento académico, donde plasma la necesidad del desarrollo de complejas habilidades, actitudes y valores que deben estar contenidas en la formación de cualquier docente para poder provocar aprendizajes significativos y competencias adecuadas en los estudiantes.

Según Campoverde (2014): .. "Las Competencias Docentes, no sólo implican el uso de técnicas, estrategias y metodologías, que él o la docente hace suyas y las lleva al aula; hace referencia a una extensa comprensión sobre un cuerpo de valores, creencias y formas de hacer las cosas, las consideran como una práctica social, compleja, incierta, imprevisible, dinámica, contextualizada y condicionada por factores de tipo político, económico y cultural, así como por factores como la formación previa, la experiencia, las expectativas y la motivación".

En este mismo año (Espinoza, 2014), declara que..." Un profesional de la educación es competente en lo que respecta a la metodología de enseñanza cuando comunica ideas y conceptos con claridad en los diferentes ambientes de aprendizaje y ofrece ejemplos pertinentes a la vida de los estudiantes".

Para (Alonso, 2020),.. "Las competencias docentes, implica asumir con responsabilidad los retos de la educación frente al comportamiento conductual de los estudiantes, con su habilidad y destreza que la profesión exige, debiendo mantener prudencia paciencia para abordar y modificar las necesidades de los estudiantes debiendo ser práctica en el interior del salón de clase con el objetivo de controlar las conductas disruptivas presentes."

(Flores, 2018), al referirse a la capacitación como un proceso a través del cual se adquieren, actualizan y desarrollan conocimientos, habilidades y actitudes para el mejor desempeño de una función o conjunto de ellas. Se basa en las necesidades o requerimientos de un grupo de personas, en este caso de la comunidad educativa. 


\section{Metodología}

La investigación fue desarrollada en la Unidad Educativa “Adalberto Ortiz Quiñonez", ubicada en la Cooperativa. Nueva Prosperina de Guayaquil. Basada en una metodología mixta, con diseño no experimental, de tipo descriptiva, documental y bibliográfica. Como parte de los métodos teóricos empleados se encuentran el histórico-lógico, el analíticosintético, el inductivo -deductivo y el sistémico - estructural funcional y como método empírico se utilizó la observación, ambos grupos de métodos estuvieron acompañados de instrumentos de investigación tales como la encuesta, entrevista, ficha de observación y el grupo focal.

La muestra fue seleccionada de forma directa de tipo no probabilística e intencional, considerando 2 directivos, 7 docentes, 20 padres de familia y 20 estudiantes para un total de 49 participantes correspondiente al 51,09\% de la población total

\section{Análisis y Discusión de Resultados}

Del análisis de los instrumentos aplicados resultaron muy significativos los obtenidos en la encuesta aplicada a estudiantes, la cual estuvo integrada por 11 preguntas, destacándose aquí la necesidad de buscar una solución viable para el desarrollo de las competencias docentes que permitan atender oportunamente a los estudiantes con conductas disruptivas

Obsérvese como en la pregunta 3, referente a: qué hace el docente cuando presencia una acción de comportamientos problemáticos?. Quedó determinado que existe un alto porcentaje de docentes, correspondiente a un $40 \%$ que no prestan atención cuando en el aula se presentan comportamientos problemáticos por parte de los estudiantes. Esta percepción de los estudiantes debe ser tenida en cuenta para dar solución a los conflictos que se presenten durante las actividades escolares.

Tabla 1 Comportamientos problemáticos

\begin{tabular}{lcc}
\hline \multicolumn{1}{|c}{ Alternativas } & Cantidad & Porcentaje \\
\hline $\begin{array}{l}\text { Llama la } \\
\text { atención }\end{array}$ & 4 & 20 \\
$\begin{array}{l}\text { Busca al } \\
\begin{array}{l}\text { Inspector } \\
\text { No le presta } \\
\text { atención }\end{array}\end{array}$ & 4 & 20 \\
$\begin{array}{l}\text { Soluciona el } \\
\text { conflicto } \\
\text { Total }\end{array}$ & 4 & 40 \\
\hline
\end{tabular}

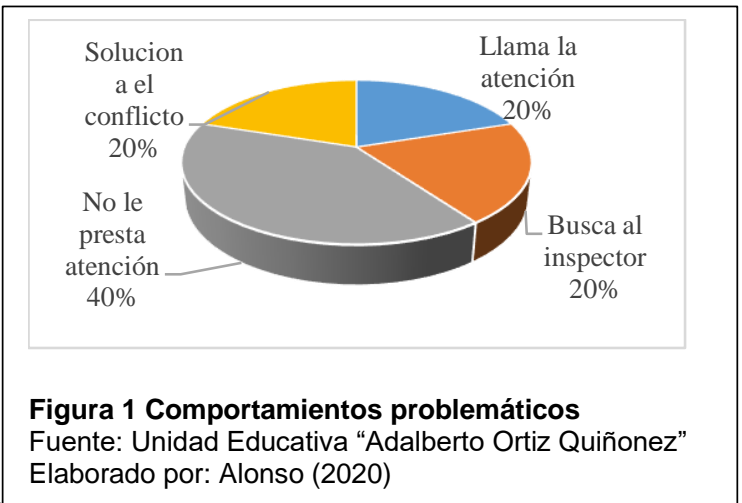

Fuente: Unidad Educativa "Adalberto Ortiz Quiñonez"

Elaborado por: Alonso (2020)

Otra de las preguntas que llama la atención es la número 7 relacionada con la utilización de estrategias pedagógicas por parte de los docentes, los resultados obtenidos permiten interpretar que un $45 \%$ de los docentes no utilizan estrategias pedagógicas para mantener el control disciplinario en las actividades escolares, por lo tanto, la percepción del 
estudiante es que los docentes deben aplicar estrategias pedagógicas o didácticas frente a los actos de indisciplinas y en mantener el control de los comportamientos en la clase.

Tabla 2 Utiliza estrategias pedagógicas

\begin{tabular}{lcc}
\hline Alternativas & Cantidad & Porcentaje \\
\hline Si & 4 & 20 \\
No & 9 & 45 \\
No se & 7 & 35 \\
Total & $\mathbf{2 0}$ & $\mathbf{1 0 0 \%}$
\end{tabular}

Fuente: Unidad Educativa "Adalberto Ortiz Quiñonez" Elaborado por: Alonso (2020

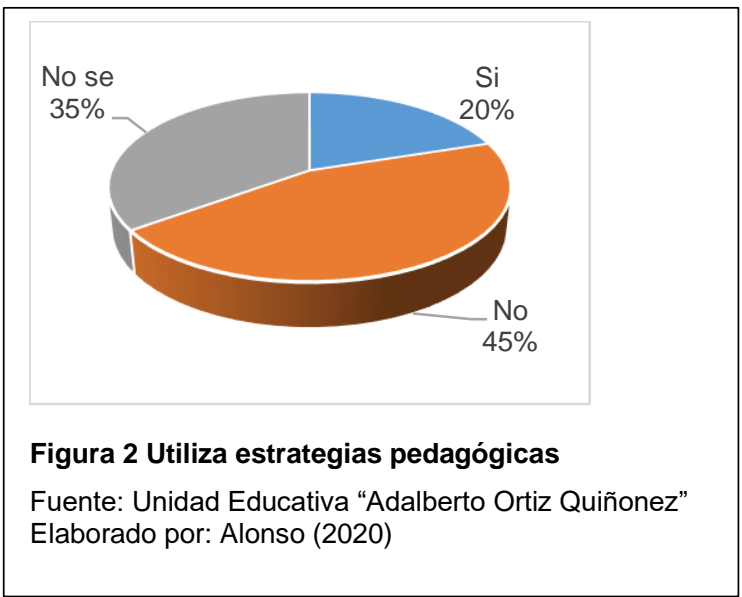

Los resultados de la entrevista aplicada a los directivos también enfatizan en la necesidad de optimizar las competencias docentes para solucionar los problemas de conductas disruptivas en los estudiantes. Obsérvese las respuestas dada por los dos directivos entrevistados a las preguntas 2, 4 y 7 que conforman la entrevista aplicada

\section{2. ¿Cómo es el accionar de los docentes para atender las conductas disruptivas?}

$\mathrm{R} 1=$ Por lo general, el docente informa sobre conductas disruptivas de los estudiantes, sean graves o no y que no las puede solucionar en el salón de clase.

$\mathrm{R} 2$ = En ocasiones los docentes acuden a la autoridad superior para informar sobre eventos inapropiados de los estudiantes, con el fin de atenderla solución.

4. ¿Cree Ud. que las manifestaciones de conductas en el aula, afecten las competencias docentes?

$\mathrm{R} 1=$ Las manifestaciones de estas conductas disruptivas afectan notablemente porque no hay respeto al entorno de aprendizaje por parte de los estudiantes, sin embargo, el docente debe mantener un autocontrol, debe entender estas conductas e intentar mantener la calma y no levantar el tono de voz.

$\mathrm{R} 2$ = Es necesario que el estudiante perciba la seguridad del docente, para imponer una medida de corrección en un tono firme que no amenace la integridad del estudiante, esta habilidad y competencia del estudiante debe mostrarla en el día a día de la jornada escolar.

7. ¿Piensa Ud. que si se efectuar un manual de actividades para los docentes mejoraría las conductas disruptivas de los estudiantes?

R1= Es importante la ejecución de actividades que tengan un contenido de motivación y dinámicas donde se utilice el humor, para generar un ambiente distendido en el interior del salón de clase. 
R2 = Es positivo poseer un manual de actividades con contenido en dinámicas para que los docentes puedan ejecutar, siendo una guía didáctica que permita buscar reducir las conductas disruptivas, usando un refuerzo en el respeto entre los estudiantes.

\section{Análisis de los resultados de la ficha de observación aplicada de los docentes}

En el análisis de los resultados obtenidos mediante la aplicación de la ficha de observación áulica, permitió examinar el grado competencia de los docentes para atender a los estudiantes con conductas disruptivas, de la Unidad Educativa "Adalberto Ortiz Quiñonez", sustentado la pertinencia y factibilidad para la aplicación de la propuesta, quedando justificada con el análisis de lo observado en el interior del salón de clases.

Los resultados obtenidos tuvieron tres momentos:

- Sobre el abordaje de las conductas disruptivas.

- Convivencia escolar

- Voluntad y Actitud para la atención de conductas disruptivas.

Destaca en la observación realizada que los docentes aun desarrollan actitudes y habilidades que caen en la educación tradicional, impidiéndose cumplir con los objetivos de la clase, entre otros aspectos porque tienen que abordar las conductas disruptivas que presentan los estudiantes. Requiriendo desarrollar estrategias metodológicas adecuadas que permitan dar solución a esta problemática, ya que existe un porciento elevado de los docentes participantes que en muy pocas ocasiones aplican alguna estrategia pedagógica y metodológica que dé respuesta al control de la situación en las conductas disciplinarias durante la jornada escolar.

De igual manera es muy preocupante que nunca generan un ambiente participativo y armónico para el control de las conductas disruptivas. En la aplicación de acciones de sensibilización para las conductas disruptivas los resultados muestran que resulta muy escasa la aplicación de estas técnicas como alternativas de compensación conductual, existiendo limitaciones en cuanto al desarrollo de formas participativas o el desarrollo de actividades que generen un clima de confianza, sentido de pertenencia y formación de cultura de paz, unida a una promoción de detección temprana, remisión y solución de conflictos. Si resulta significativo que reflejan que tienen motivación para conocer y ejecutar actividades que modifiquen las conductas disruptivas.

\section{Análisis de la aplicación del grupo focal con la participación de los docentes}

Durante la realización del grupo focal aplicado a los docentes sobre las conductas disruptivas. Esencialmente se interpreta lo siguiente:

$\checkmark$ Se debe aplicar una estrategia de intervención en el proceso educativo, considerando el poder de trasmisión y construcción del conocimiento como una forma de apoyo para el aprendizaje, que fortalezcan las relaciones entre los estudiantes.

$\checkmark$ Se presentan con mayor frecuencia las conductas disruptivas al iniciar peleas entre ellos, molestar deliberadamente a otros estudiantes, actitudes de desobediencia, 
interrumpir cuando habla el docente, levantarse frecuentemente de su asiento, dificultad para esperar, no seguir las instrucciones de los docentes.

$\checkmark$ Sobre si conocen las competencias docentes para la atención de las conductas disruptiva, se manifestó que muchas veces las competencias están condicionados a los factores internos y externos, y que requieren de orientaciones precisas con la finalidad de poder asesorar, dar soporte, ofrecer recursos, orientaciones y acciones para mejorar su atención, de una manera coordinada y proactiva durante la jornada escolar.

$\checkmark$ Sobre el diseño de manual de actividades que contengan estrategias metodológicas que mejore la atención de conductas disruptivas de los estudiantes, se coincide que es importante su aplicación o ejecución para mantener un estudiante motivado y dedicado a sus actividades escolares, con el propósito de disminuir los altos índices de conductas disruptivas.

\section{Discusión de resultados}

Como se puede apreciar es coincidente que en todos los instrumentos aplicados durante el proceso investigativo tanto los estudiantes como los directivos y docentes participantes, señalen la importancia del desarrollo de competencias docentes para la atención dentro del salón de clases de los dicentes con conductas disruptivas, todo esto reafirma la idea inicial de la investigación referente al diseño de un Manual de Actividades Lúdicas como alternativa viable a ser utilizada por los docentes

Quedando así confirmado la necesidad de intervenir y provocar estrategias que promuevan las acciones de prevención durante la jornada escolar y que ayuden a mejor la atención de las conductas disruptivas de los estudiantes durante la jornada escolar, esto nos hace coincidir con los aportes realizados por Morazán (2013), (Espinoza, 2014), (Campoverde, 2014), (Flores, 2018), quienes en sentido general señalan la valía e importancia que tiene para el proceso de inclusión educativa de los estudiantes con conductas disruptivas y su posible corrección u compensación el desarrollo de las competencias docentes, ya que el profesor se convierte en el apoyo, facilitador y ejemplo a seguir por parte de los estudiantes. Por otra parte es fundamental significar la necesidad de tener un abordaje multidisciplinar que incluya la participación del medio familiar y por consiguiente la inclusión y participación del propio dicente, quien debe participar de manera consciente en la corrección de su conducta y como es lógico pensar todo esto implica un desafío permanente para lo cual el docente, el contexto socio familiar y educativo debe estar preparado.

\section{Propuesta}

Los resultados obtenidos permitieron realizar y fundamentar la propuesta, la que consistió en diseñar un Manual de Actividades lúdicas para docentes conducente a la atención de conductas disruptivas, la misma fue validada mediante el criterio de especialistas, contando con el $100 \%$ de aprobación. 
El Manual diseñado contiene un sistema de actividades y estrategias metodológicas que ayudan a fortalecer las competencias docentes, mejorando los procesos de atención, en la misma medida que van disminuyendo las conductas disruptivas de los estudiantes durante la jornada escolar en el salón de clase.

El mismo está compuesto por: Introducción, justificación, objetivo general y específicos, recursos materiales y humanos, limitaciones, alcance, cronograma y beneficios.

La estructura seguida permitió integrar un total de 19 actividades (juegos) los cuales se distribuyen como sigue:

\section{Gráfico Número 3 Esquema Representativo del Manual de Actividades.}

\section{ESQUEMA REPRESENTATIVO DEL MANUAL DE ACTIVIDADES}

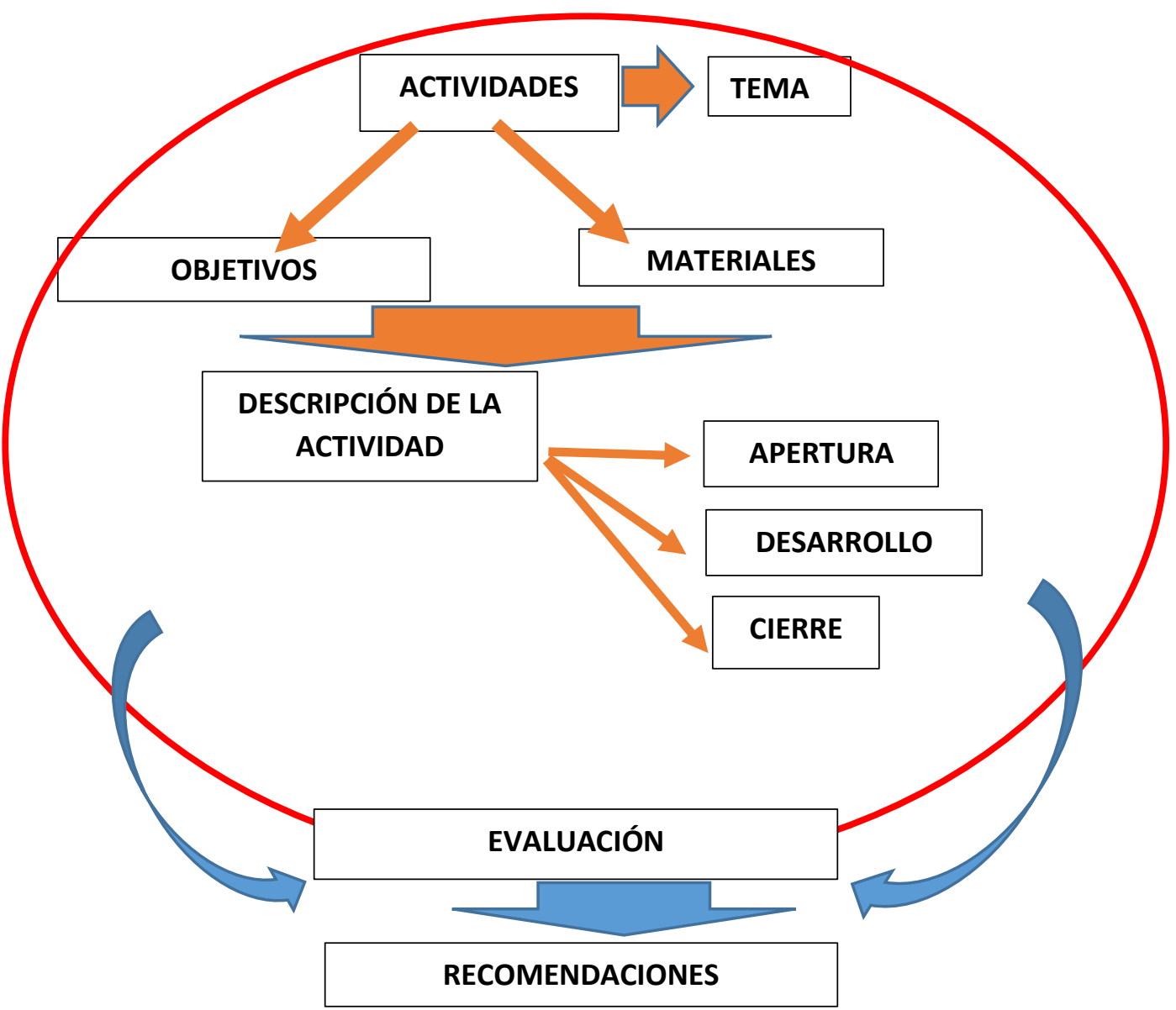

\section{Conclusiones}

- El análisis de los fundamentos teóricos y la constatación de las evidencias empíricas planteadas, permiten afirmar que existe una contradicción entre la manifestación de conductas disruptivas en el salón de clases por algunos estudiantes muestra de estudio y la preparación que tienen los docentes para enfrentar con éxito la atención a estas necesidades educativas. 
- Lo anterior confirma la idea inicial de la investigación, quedando demostrado la necesidad de abordar este tema de manera multidisciplinar con énfasis en la participación de todos los implicados. Todo esto supone un desafío permanente para lo cual el docente, el contexto socio familiar y educativo debe estar preparado.

- La elaboración y aplicación del Manual de actividades lúdicas contribuirá a dar solución a la problemática estudiada, ya que los docentes de la Unidad Educativa de referencia podrán contar con una herramienta de apoyo y de gran utilidad para mejorar su desempeño y competencias docentes en beneficio de la comunidad educativa.

\section{Referencias Bibliográficas}

Alonso, G. (2020), Competencias del docente en la atención a estudiantes con conductas disruptivas de 8vo año de Educación General Básica. Tesis en Opción al grado de Magister en Inclusión Educativa y Atención a la Diversidad Universidad Universidad Laica Vicente Rocafuerte. Guayaquil. ( próxima a sustentación)

Campoverde, M. (2014). Desempeño docente en el proceso de enseñanza aprendizaje en el nivel de Educación Básica de la Unidad Educativa "Belisario Quedo" en la ciudad de Pujilí, provincia de Cotopaxi, Año lectivo 2013-2014. Cotopaxi-Ecuador: Universidad Técnico Particular de Loja.

Cépedes, A. (2012). Niños en Pataletas.adolescentes desafiantes, Como manejar los trastornos de conductas en los hijos.Santiago de Chile: Ediciones Básicas Chile SA

Cuberes, R (2013) Causas de las conductas desafiantes en alumnos de primaria.Trabajo de fin de Grado Universidad Internacional de la Rioja.Facultad de Educación.

Duarte, J. (2017). Formación permanente de docentes en servicio, alternativa para la enseñanza y el aprendizaje de la lengua escrita en la educación básica integral. México: Universidad Rovira.

Freire, C. (2012). Factores que inciden en la presencia de conductas disruptivas de los estudiantes de $\operatorname{los} 8^{\circ}, 9^{\circ} 10^{\circ}$ años en educación básica de la Unidad Educativa experimental FAE Nº3 Taura del Cantón Yaguachi durante el período lectivo 2012. Milagro: Universidad Estatal de Milagro.

Flores, G. (2018). Programa de capacitación psicológica a padres de familia para la atención de niños con conductas disruptivas de 6to EGB. Tesis en opción al grado de Magister en Inclusión Educativa y Atención a la Diversidad. Universidad Laica Vicente Rocafuerte. Guayaquil.

Espinoza, G. (2014). Desempeño docente en el proceso de enseñanza aprendzaje en el nivel de Educación Básica Superior del Centro Educativo Colegio de Bachillerato Ciudad de Portovelo. Loja: Universidad Técnica Particular de Loja. 
Equipo Dide, 2017, Conductas disruptivas. La asertividad, una competencia docente primordial. http://educaryaprender.es/conductas-disruptivas-comportamiento/

Expasa. (2018). Diccionario Enciclopédico. En T. I.-t. Edición-. Barcelona: Expasa Calpe.

Jiménez, J. (2017). Conductas disruptivas en el Aula y desempeño docentes en la Institución Educativa Técnica General Roberta Leyva del Municipio de Saldaña Tolima. Ibague: Universidad del Tolima.

Jurado, P. (Coord.)(2015), Informe "Influencia de los comportamientos disruptivos en el fracaso escolar de los alumnos de ESO Hacia un modelo de intervención centrado en la institución educativa” Barcelona: UAB (Proyecto I+D-2010. EDU2010-20105 (subprograma EDUC)

Jurado, P \& Justiano MD(2015), "Las conductas disruptivas y los procesos de intervención en la educación secundaria” Universidad Autónoma de Barcelona (España) Cooperación Técnica Alemana (Bolivia)

Morazán, S. (2013). Competencias docentes y su relación con el rendimiento académico en la asignatura de matemáticas en las instituciones de educación media del municipio de Danlí. Tegucigalpa: Universidad Pedagógica Nacional "Francisco Morazán". 


\section{PARA CITAR EL ARTÍCULO INDEXADO.}

Alonso Delgado, G. T., Paredes Argudo, M. J., \& Salazar Olaya, G. D. (2020). Competencias docentes y conductas disruptivas: Un desafío permanente en el contexto educativo. Ciencia Digital, 4(4.1), 18-31. https://doi.org/10.33262/cienciadigital.v4i4.1.1450

\section{Ciencia \\ LDigital}

El artículo que se publica es de exclusiva responsabilidad de los autores y no necesariamente reflejan el pensamiento de la Revista Ciencia Digital.

El artículo queda en propiedad de la revista y, por tanto, su publicación parcial y/o total en otro medio tiene que ser autorizado por el director de la Revista Ciencia Digital.
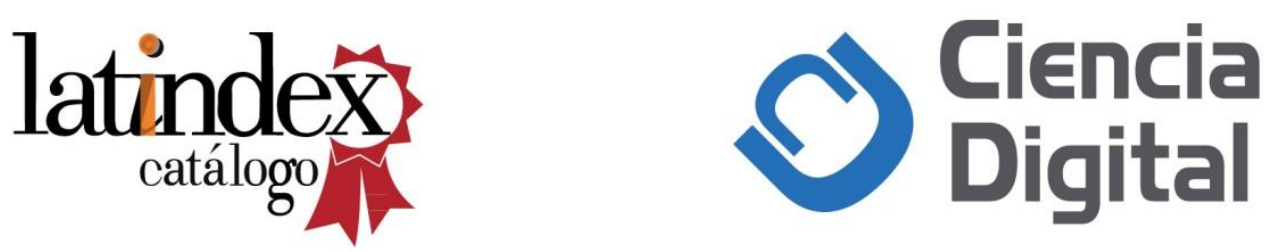